\title{
ESTUDIO PETROGRÁFICO DE LA CERÁMICA ARQUEOLÓGICA DEL PRIMER MILENIO D.C. AL SUR DE LOS VALLES CALCHAQUÍES (NOROESTE ARGENTINO)
}

\author{
A PETROGRAPHIC STUDY OF FIRST MILLENNIUM AD ARCHAEOLOGICAL \\ CERAMICS FROM SITES TO THE SOUTH OF THE CALCHAQUÍ VALLEYS \\ (NORTHWEST ARGENTINA)
}

\author{
Lucas Pereyra Domingorena ${ }^{1}$
}

\begin{abstract}
En este artículo se presenta la investigación que procuró establecer los estilos técnicos de manufactura cerámica utilizados por las alfareras y alfareros de las sociedades agropastoriles que habitaron, durante el primer milenio d.C., al sur de los valles calchaquíes (Noroeste argentino).

La metodología partió de considerar a los recipientes cerámicos como las unidades de análisis, debido a que fueron los objetos realmente usados en los contextos domésticos prehispánicos estudiados. Se implementó el estudio petrográfico de las pastas cerámicas para registrar posibles patrones mediante las diferencias y semejanzas registradas. A partir de los patrones detectados, combinando los datos experimentales y las comparaciones con las distintas litologías locales del área de estudio, se establecieron los estilos técnicos utilizados para elaborar cada uno de los recipientes reconstruidos. Así, se vincularon las maneras de hacer con el repertorio morfológico y las representaciones y diseños de distintos estilos cerámicos.

Se estudiaron 155 cortes delgados. Como resultado se determinó la existencia de una producción alfarera de carácter doméstico y local predominante, junto con la presencia, en menor frecuencia, tanto de objetos procedentes de áreas foráneas como de objetos que se asemejan a cerámicas de otras áreas del Noroeste argentino. Esto permitió comprobar la existencia de una tradición artesanal con manifestaciones locales que estuvo vigente en el área de estudio durante el primer milenio d.C.
\end{abstract}

Palabras claves: arqueología, Noroeste argentino, período Formativo, petrografía cerámica.

This article describes a research project that sought to establish technical ceramic manufacturing styles used by potters of agropastoral societies that lived during the first millennium $A D$ at sites to the south of the Calchaqui valleys (Northwest Argentina). The methodology considered ceramic containers as the units basic of analysis, as these were the objects actually used in the prehispanic domestic contexts under study. Secondly, petrographic analysis permitted the identification of possible patterns through the differences and similarities observed in the fabrics. Finally, different technical styles for each of the reconstructed vessels were inferred through a combination of petrographic and experimental results, as well as lithological data from the study area. The resulting patterns link particular 'ways of making' with specific variations in the morphological repertoire as well as with different ceramic designs and styles.

A total of 155 thin sections were studied. The study identified domestic pottery production of predominantly local character, alongside low frequencies of foreign objects, and locally produced objects that resemble ceramics from other areas of NW Argentina. This allowed verifying the existence of a craft tradition with local manifestations that prevailed in the study area during the first millennium $A D$.

Key words: Archaeology, Northwest Argentina, Formative Period, ceramic petrography.

El objetivo de mi investigación fue establecer los antiguos estilos técnicos de manufacturas cerámicas utilizados por las alfareras y alfareros de las sociedades agropastoriles que habitaron al sur de los valles calchaquíes durante aproximadamente todo el primer milenio d.C. Para esto se utilizaron los materiales cerámicos procedentes de ocho sitios arqueológicos: Cardonal y Bordo Marcial (sur del valle del Cajón); Ingenio Arenal-Falda del Cerro, Loma Alta, Tesoro I, Antigal de Tesoro y Morro de las Espinillas (faldeo occidental del Aconquija); y Bañado Viejo (llanura aluvional del río Santa María). Scattolin (2007a) ha establecido que las ocupaciones humanas de estos sitios tienen una cronología que

1 CONICET - Museo Etnográfico, Facultad de Filosofía y Letras, Universidad de Buenos Aires, Argentina. lucasdomingorena@gmail.com 
comienza en el siglo primero d.C. y finaliza hacia el siglo nueve d.C. Además ha concebido para este rango temporal tres fases, denominadas Chimpa (100-450 d.C.), Bañado (450-650 d.C.) y Colalao (650-900 d.C.) (Figura 1).

Con relación a la cerámica, la metodología implementada procura reconocer los recipientes, como unidades morfológicas, dentro del registro material recuperado de los sitios arqueológicos estudiados. De esta forma, la vasija es considerada como la unidad mínima del análisis, es decir, se pone en evidencia a los objetos reales usados en los contextos domésticos prehispánicos para luego estudiar la manera en que fueron confeccionados.

\section{Una Clasificación Preliminar}

Para tal fin, entonces, se buscó en primer lugar la caracterización de la alfarería a partir de una clasificación de los atributos macroscópicos de los fragmentos, es decir, los atributos de diseños, la textura general de las pastas, la coloración, los tratamientos de superficie, etc. Se utilizó una serie de tipos cerámicos definidos para el Noroeste argentino (Apéndice I en Pereyra Domingorena 2010:402-405).

Posteriormente se realizaron las reconstrucciones de forma de las vasijas. El procedimiento seguido es convencional y consiste en la estimación del diámetro de la pieza en una "carta de diámetros".

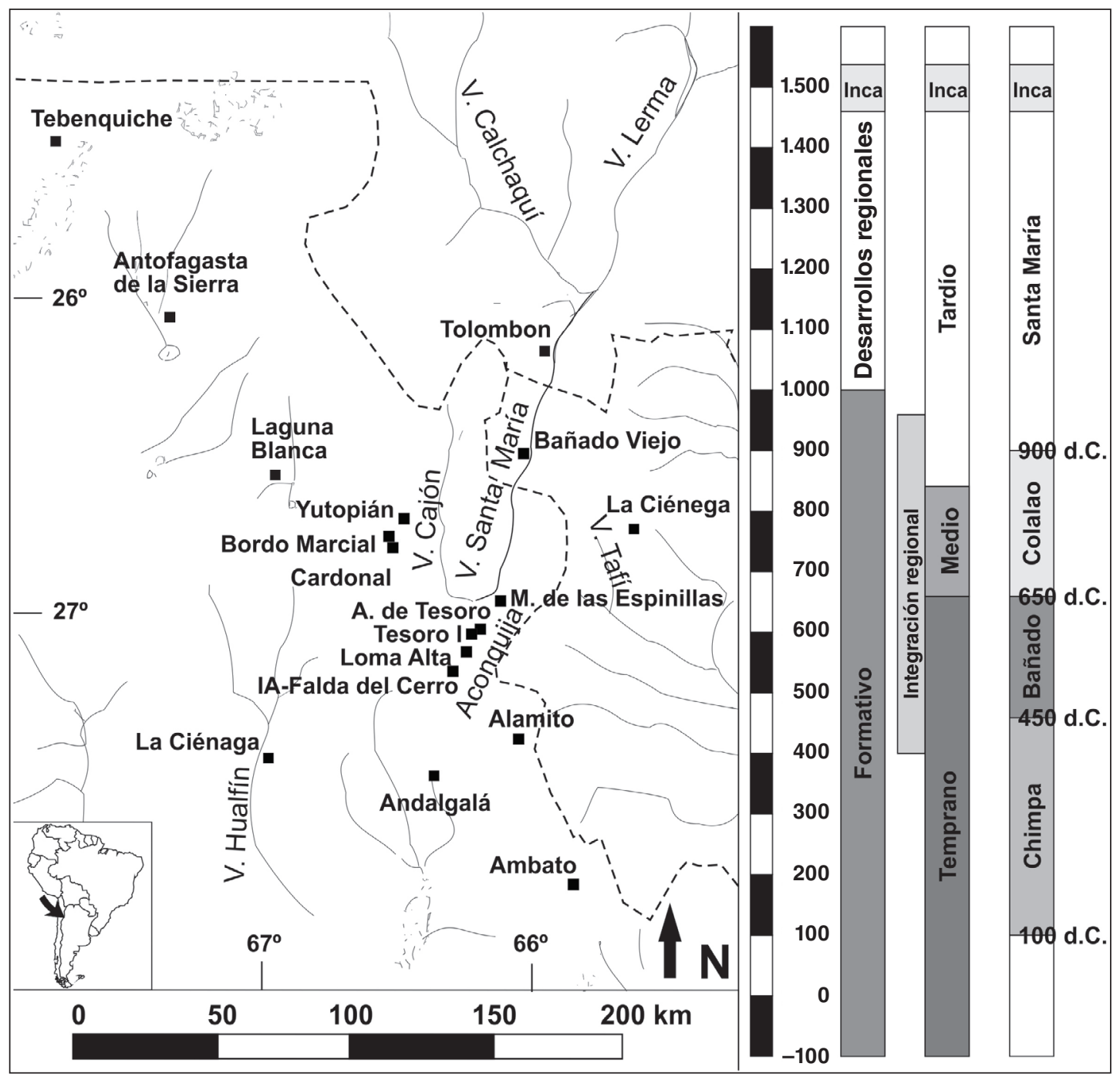

Figura 1. Área de estudio y cronología (cuadro temporal elaborado a partir de Scattolin 2007a). Study area and chronology (temporal table from Scattolin 2007a). 
Luego se dibuja la sección de la vasija a partir de la información métrica obtenida y mediante la correcta orientación del fragmento. También, de ser posible, se adicionan los tiestos de cuerpo a la misma unidad reconstruida por el fragmento de borde. Una vez obtenida la silueta reconstruida -sea con un borde o con más fragmentos de cuerpo- se asignaron categorías morfológicas a partir de mediciones simples de profundidad, diámetro máximo y diámetro de la boca (Balfet et al. 1983, 1988). Esta tipología clasificatoria general fue adaptada a las características propias de la muestra y a las posibles variantes al interior del tipo morfológico (Bugliani 2008; Scattolin 1990, 2007b). De esta manera se obtuvo el repertorio de recipientes que los pobladores de las aldeas formativas usaron a diario.

Sin embargo, debemos aclarar que otros tiestos que mostraban variaciones o características técnicas no presentes en las vasijas reconstruidas fueron considerados en el análisis. Pero además, entendemos que algunos de estos pudieron haber participado en la vida cotidiana no como recipientes sino como fragmentos en sí. En este sentido, “cada uno de esos fragmentos [podrían ser] un comentario, una nota, que diera sentido a la narración de las experiencias de los viajes" (Haber 2007:66), es decir, "fragmentos que han sido intencionalmente conservados, posiblemente como referentes de historias de relaciones, intercambios e interacción" (Bugliani y Pereyra Domingorena 2012:136).

De esta manera, se conformó una muestra de 155 ejemplares, de estas 108 representan recipientes reconstruidos parcialmente y 47 corresponden a fragmentos diagnósticos hallados en los ocho sitios arqueológicos antes mencionados (Tabla 1).

\section{El Estudio Petrográfico de las Pastas Cerámicas}

Una vez seleccionada la muestra $(\mathrm{n}=155)$ se procedió a realizar la caracterización petrográfica de las pastas cerámicas por la descripción de las secciones delgadas (Figura 2). Estas fueron analizadas en un microscopio de luz polarizada Leica modelo DME-P y fotografiadas con una cámara digital CANON Power Shot S80 en el objetivo de 5x/0,12, lo que permite obtener una imagen óptima del corte.

El procedimiento petrográfico se desarrolla en cuatro etapas. En la primera se describe el fondo de pasta, es decir, la matriz arcillosa y los elementos no plásticos menores a $15 \mu \mathrm{m}$ (Courtois 1976; Cremonte 1996). Este fondo se caracteriza según la coloración y las distintas estructuras (pseudolepidoblástica, microgranosa y/o criptofilitosa, según las distinciones fijadas por Courtois 1976). Se estableció el tamaño mínimo de las inclusiones no plásticas en $15 \mu \mathrm{m}$, debido a que las inclusiones menores a este límite no pueden ser identificadas petrográficamente de forma precisa. En consecuencia, se determinaron los elementos propios de la materia prima arcillosa y los elementos no plásticos.

La segunda etapa consiste en la determinación mineralógica $^{1}$ de las inclusiones mayores a 15 $\mu \mathrm{m}$. Estas se clasificaron en cristaloclastos, es decir, fragmentos de cristales individuales como cuarzo, feldespato potásico, biotita, turmalina,

Tabla 1. Cantidad de vasijas reconstruidas y muestras fragmentarias analizadas petrográficamente. Number of vessels reconstructed and fragmentary samples analyzed petrographically.

\begin{tabular}{|c|c|c|c|c|c|}
\hline \multirow[b]{2}{*}{ Sitio } & \multicolumn{3}{|c|}{ Vasijas } & \multirow[b]{2}{*}{ Fragmentos } & \multirow[b]{2}{*}{ Total } \\
\hline & $\begin{array}{c}\text { Cántaros } \\
\text { y Ollas }\end{array}$ & $\begin{array}{c}\text { Jarras, jarros } \\
\text { y vasos }\end{array}$ & $\begin{array}{l}\text { Cuencos y } \\
\text { escudillas }\end{array}$ & & \\
\hline Cardonal & 4 & 2 & 1 & 6 & 13 \\
\hline Bordo Marcial & 0 & 0 & 0 & 4 & 4 \\
\hline IA-Falda del Cerro & 2 & 0 & 0 & 6 & 8 \\
\hline Loma Alta & 21 & 15 & 19 & 7 & 62 \\
\hline Tesoro I & 3 & 2 & 2 & 0 & 7 \\
\hline Antigal de Tesoro & 0 & 3 & 4 & 9 & 16 \\
\hline M. de las Espinillas & 6 & 0 & 5 & 2 & 13 \\
\hline Bañado Viejo & 7 & 2 & 10 & 13 & 32 \\
\hline Total & 43 & 24 & 41 & 47 & 155 \\
\hline
\end{tabular}




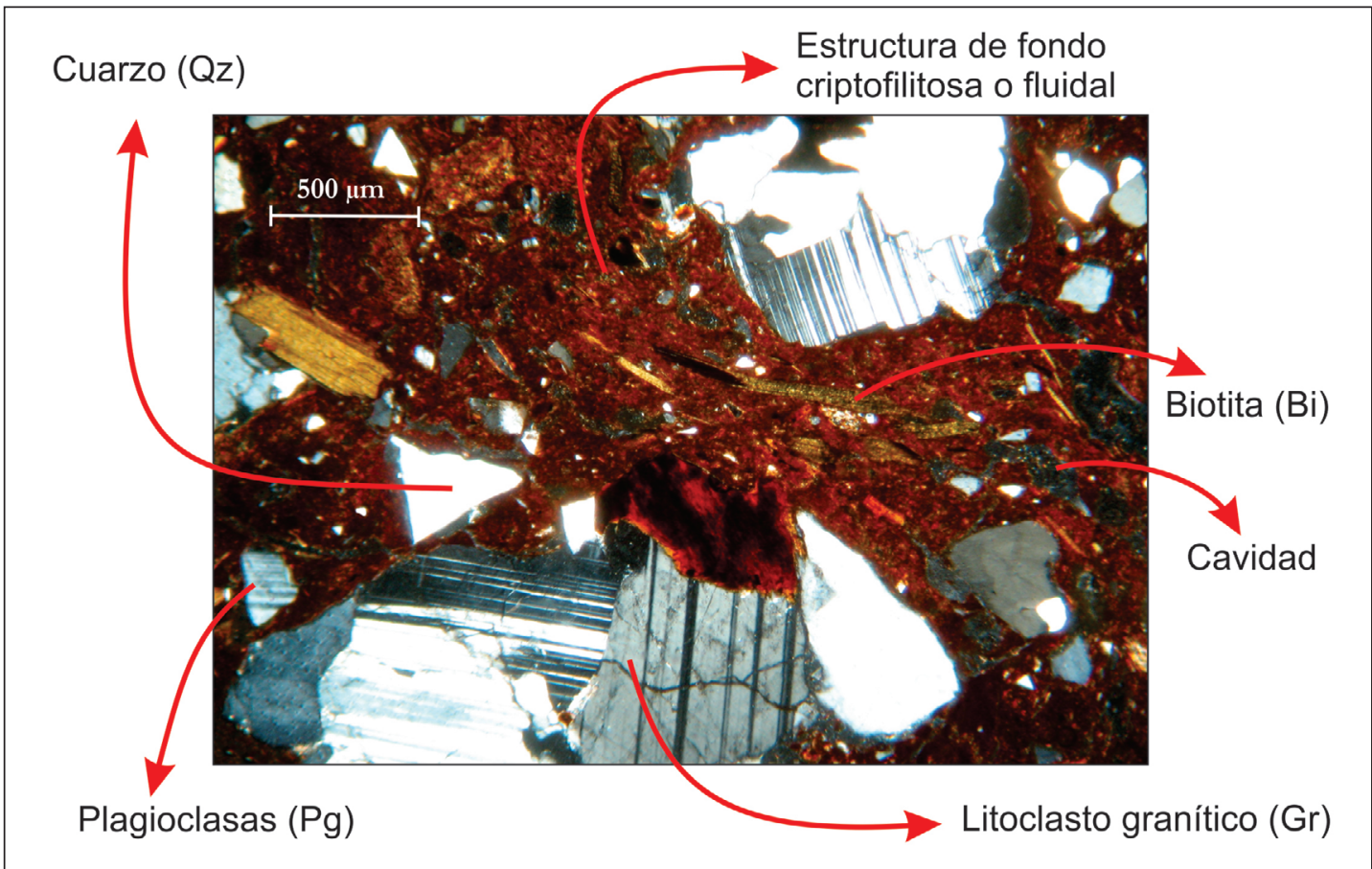

Figura 2. Ejemplo de una pasta cerámica y sus atributos.

Example of ceramic fabric and its attributes.

entre otros. También se detectaron litoclastos, o sea, fragmentos de rocas, los que pueden tener un origen ígneo (volcánico o plutónico), sedimentario o metamórfico. Además se observaron otros elementos como minerales opacos, vidrio volcánico, gránulos de arcilla y tiesto molido. Según sus hábitos y formas, los distintos clastos fueron clasificados en tabulares, laminares, angulares, subangulares, subredondeados o redondeados (Cremonte 1996). Para los tamaños de las inclusiones se utilizó la escala granulométrica de Wentworth (tomada de Adams et al. 1997 [1984]).

En la tercera fase se observaron y clasificaron las cavidades (poros y oquedades) presentes en las pastas cerámicas. Se registró su abundancia, tamaños y formas. La importancia de las cavidades se relaciona con el proceso de amasado y uso del recipiente (Cremonte 1996; Linné 1925). Las formas fueron clasificadas en redondeadas, alargadas o irregulares.

La cuarta y última fase consistió en el cálculo porcentual de la distribución modal de los componentes que integran la pasta (matriz, inclusiones y cavidades). Para ello se efectuó un conteo mínimo de 300 puntos (point counter) por corte delgado. Durante esta etapa, además, se registraron las formas y tamaños tanto de las inclusiones no plásticas como de las cavidades, para ello se utilizó la escala micrométrica del microscopio.

Por medio de un análisis comparativo de los registros de las pastas cerámicas y del empleo del método de conglomerados (cluster analysis) ${ }^{2}$ se elaboró una tipología basada en las características petrográficas cualitativas (estructuras de fondo, tamaño y forma tanto de las inclusiones como de las cavidades) y cuantitativas (porcentaje de matriz, cavidades y los distintos tipos de inclusiones registrados). Posteriormente, los tipos cerámicos fueron correlacionados con otros atributos de la cerámica -morfología, tipo de cocción, representaciones plásticas- para evaluar posibles modalidades de manufacturas alfareras del período estudiado y para plantear hipótesis sobre procedencia a partir de comparaciones entre la mineralogía observada y la litología de la zona donde se emplazan los sitios arqueológicos.

\section{Estilos Técnicos}

En suma, el procedimiento implementado permitió caracterizar la pasta cerámica a partir de 
la descripción de las estructuras de fondo de la matriz arcillosa y la cuantificación e identificación tanto de los materiales no plásticos como de las cavidades. Se consiguió de esta manera una clasificación petrográfica independiente de otras catalogaciones de la cerámica, ya sea por morfología o por decoración (Culbert y Rands 2007). Pero al mismo tiempo se podía vincular un tipo de pasta con una silueta particular, es decir, que no se creaba solo una tipología de pastas -aislada de las unidades reales, las vasijas-, sino que se podía saber el modo por el cual se había logrado una determinada olla.

El examen de las pastas permitió inferir algunos comportamientos técnicos. En primer lugar, el agregado intencional de atemperante se manifestó en la distribución bimodal, tipo y cantidad de material no plástico; la comparación textural de gránulos de arcilla y el fondo de pasta; y también, en la comparación con cerámicas coetáneas sin inclusiones mayores de $15 \mu \mathrm{m}$ y patrones cerámicos experimentales (ver más adelante). En segundo lugar se pudo estimar la calidad del amasado mediante las formas y densidad de cavidades; la orientación de estas y de las inclusiones no plásticas. Finalmente, se monitorearon las atmósferas y temperaturas de cocción, tanto por las texturas y colores de las pastas como por la comparación con patrones experimentales (Cremonte 1996; Pereyra Domingorena 2010; Puente 2011).

A partir de la evaluación del ordenamiento estadístico de los datos petrográficos y análisis comparativo de las diversas combinaciones de los atributos de las pastas -las estructuras de la matriz, las inclusiones y las cavidades- se definieron los estilos técnicos registrados en la investigación. En este sentido, entendemos que estos estilos son una combinación particular de las materias primas, las fuentes de energía ${ }^{3}$, las herramientas y la planificación (Stark 1999), pero además de habilidades incorporadas y conocimiento práctico (Lemonnier 1992; Mauss 1979 [1935]), todos ellos elementos necesarios puestos en acción por las artesanas y artesanos para la fabricación de artefactos, es decir, que como en cualquier otra actividad técnica, la fabricación de cerámica se compone de una cadena organizada de "acciones situadas", en la que el conocimiento se pone en práctica en un entorno social y ambiental específico (Gosselien 2008:77).

En términos concretos de este estudio se identificaron siete estilos técnicos, uno representado por las pastas gruesas y sus variaciones locales; tres correspondientes a pastas medias; y dentro de las pastas finas se pudieron identificar dos estilos técnicos. Finalmente, un séptimo estilo reunió a todas las muestras de la alfarería Vaquerías, asociado a una producción alóctona al área de estudio (ver Tabla 12 en Pereyra Domingorena 2010:363).

\section{Pastas gruesas}

En primer lugar se determinó un estilo de pastas gruesas que se caracterizan por tener estructuras de fondo pseudolepidoblásticas y criptofilitosas con inclusiones con amplio rango granulométrico $(15 \mu \mathrm{m}$ a $4.000 \mu \mathrm{m}$ ), que representan entre $32 \%$ y $46 \%$ de la pasta. Es decir, que pudieron haberse empleado arenas poco seleccionadas como atemperante (Figura 3a). $\mathrm{Al}$ interior de este conjunto se distinguieron tres producciones locales que son congruentes con las variaciones petrográficas observadas y las correlaciones con las litologías circundantes a las áreas de los sitios arqueológicos. La primera reúne la alfarería ordinaria de la falda occidental del Aconquija. Estas pastas contienen abundantes cristaloclastos de cuarzo y litoclastos graníticos que constituyen entre $63 \%$ y $93 \%$ del total de las inclusiones. Esta asociación petrográfica coincide con la litología local, la que se comporta como un ambiente de rocas ígneas intrusivas ácidas (granitos, granodiorita, etc.) propias del batolito granítico que caracteriza el faldeo occidental del Aconquija (González Bonorino 1951), cuya meteorización origina arenas con las características descritas (Pereyra Domingorena 2010, 2013).

La segunda producción abarca las cerámicas del sitio Bañado Viejo, es decir, quizá una modalidad técnica del valle de Santa María. Se distingue por sus cristaloclastos de cuarzo, litoclastos graníticos, esquisto y arenisca que conforman entre 78 y $86 \%$ del total de las inclusiones. Sostenemos que existió una producción local para estos recipientes, ya que los materiales arenosos -de granulometría y composición muy variadas- son esperables en los sedimentos de una cuenca aluvional como la del río Santa María. Tal asociación material es propia de las acciones erosivas que afectaron las cumbres calchaquíes y la sierra del Cajón, de las cuales provendrían el material granítico y el esquisto; en tanto la arenisca debe proceder de las rocas sedimentarias de origen continental que integran el Grupo Santa María del Terciario (Galván 1981; Ruiz Huidobro 1972). Postulamos que la variedad 


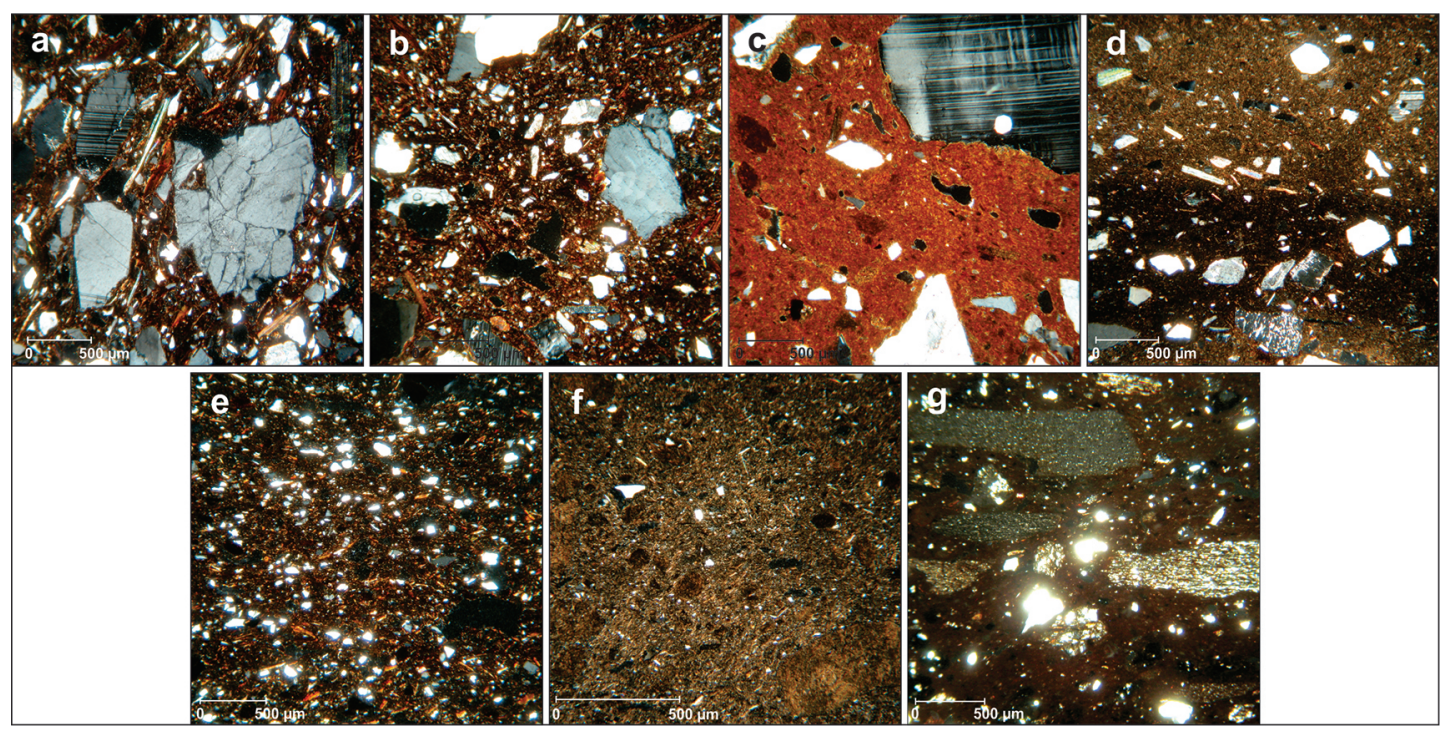

Figura 3. Ejemplos de los tipos de pastas: (a) pasta gruesa; (b) pasta media con arenas no seleccionadas; (c) pasta media con arenas seleccionadas gruesas; (d) pasta media con arenas seleccionadas medias; (e) pasta fina con agregado de arenas finas; (f) pasta fina sin agregado de arenas; (g) pasta Vaquerías.

Examples of ceramic fabric types: (a) coarse-grained paste; $(b)$ intermediate paste with no selected aplastics; (c) intermediate paste with coarse-grained selected aplastics; $(d)$ intermediate paste with medium-sized selected aplastics; (e) fine paste with aplastics; ( $f$ ) fine paste without aplastics; ( $g$ ) Vaquerías paste.

de ambientes geológicos de estas formaciones es la causa de la heterogeneidad litológica reflejada en las inclusiones de esta modalidad de pastas gruesas.

Finalmente, la tercera producción aglutina las vasijas halladas en el sitio Cardonal, es decir, lo que consideramos un modo de hacer del área del valle del Cajón (Pereyra Domingorena 2010, 2012). Su particularidad diagnóstica es la presencia de litoclastos graníticos migmatíticos y cristaloclastos de cuarzo con extinción ondulante en elevadas proporciones $(70 \%$ a $79 \%)$ respecto del total de las inclusiones. Esta asociación mineralógica es típica de un ambiente geológico de metamorfismo de alto grado. Tal fenómeno es propio de la sierra de Chango Real, a cuyos pies se encuentra localizado el sitio Cardonal. La sierra está constituida por la Formación Chango Real, integrada precisamente por granitos migmatíticos (Turner 1973). Por lo tanto, la litología del ambiente geológico es coincidente con las arenas posiblemente usadas en la preparación de las pastas cerámicas, permitiendo sustentar la hipótesis de la producción local de esta alfarería.

El estilo de las pastas gruesas puede entenderse como un "modo de hacer", que se asemeja al tipo La Ciénega Castaño Grueso y la variedad La Ciénega Castaño Pulido Grueso, descritos para la alfarería de la quebrada de La Ciénega (Cremonte 1996). Se piensa además que las similitudes petrográficas y texturales del Tipo de Pasta B1 del oasis de Laguna Blanca (Espiro Elsesser 2006) amerita su incorporación a esta manera compartida que varias sociedades aldeanas mantuvieron en uso durante el primer milenio d.C.

\section{Pastas medias}

Las pastas medias conforman un grupo heterogéneo tanto por las diferencias texturales y petrográficas registradas así como por la variedad de morfologías y tipos cerámicos que abarca. A lo largo de la secuencia histórica analizada se han podido detectar tres estilos técnicos que se distinguen por la variación granulométrica y las estructuras de fondo de pasta.

El primero se caracteriza por presentar inclusiones con un amplio rango granulométrico (30 $\mu \mathrm{m}$ a $2.000 \mu \mathrm{m}$ ), es decir, las artesanas y artesanos habrían usado arenas poco seleccionadas como atemperante, que representan entre $18 \%$ y $33 \%$ del total de la pasta. Además se describen estructuras de fondo pseudolepidoblásticas (Figura 3b). Se detectaron tres modalidades al interior de este estilo 
técnico. El análisis comparativo entre las vasijas encontradas en la falda occidental del Aconquija con estas pastas medias presenta similitudes petrográficas con las alfarerías gruesas de este sector del área de estudio (ver descripción anterior). Por lo tanto, se propone que estas pastas serían una modalidad de manufactura local. En cambio, otra modalidad estaría asociada a una producción local del valle de Santa María, ya que estas pastas medias contienen cuarzo, granitos y esquisto (60\% a $83 \%$ del total de las inclusiones), semejantes a las pastas gruesas de los recipientes hallados en el sitio Bañado Viejo. Por último, una única pasta de un recipiente del sitio Cardonal registra tanto litoclastos graníticos alterados como seudomorfos de muscovita, convirtiéndose en una pasta que no ha sido hallada en otras piezas analizadas en toda la muestra estudiada. Por las similitudes del diseño plástico de la vasija con ejemplares hallados en Tebenquiche y Laguna Blanca (Pereyra Domingorena 2010:90), postulamos que quizá esta modalidad sería común en el área de la Puna catamarqueña.

Pastas técnicamente semejantes a las descritas aparecen en zonas aledañas a nuestra área de estudio. Podrían ser comparables con el Tipo 8 de la Pasta B2 y el Tipo 6 de la Pasta C del oasis de Laguna Blanca (Espiro Elsesser 2006), y con La Ciénega Castaño Medio de la quebrada de La Ciénega (Cremonte 1996). Por lo tanto, podemos observar que estas tres modalidades serían variantes locales de un mismo estilo técnico que involucró el uso de inclusiones de variados tamaños que le proporciona a la pasta su textura media.

El segundo reúne pastas medias que se definen porque las inclusiones son gruesas $(500 \mu \mathrm{m}$ a 2.000 $\mu \mathrm{m})$, es decir, postulamos que se habría usado una arena seleccionada como material no plástico que representa entre $17 \%$ y $30 \%$ de la pasta. Las estructuras de fondo registradas son criptofilitosas (Figura 3c). Este estilo está presente en toda la secuencia temporal analizada, pero se encuentra en baja proporción $(3,65 \%)$. Este grupo de pastas se asemeja técnicamente con el tipo El Pedregal Blanco Pulido reconocido en material arqueológico hallado en la quebrada de La Ciénega (Cremonte 1996). Las diferencias petrográficas permiten postular la existencia de dos modalidades que sugieren que el conocimiento práctico para estas pastas fue adaptado a los materiales disponibles en el valle de Santa María (cuarzo, litoclastos graníticos, esquisto y arenisca) y la sierra del Aconquija (cuarzo y litoclastos graníticos) (Pereyra Domingorena 2010, 2012), habiendo quizá existido otra modalidad para la quebrada de La Ciénaga (Cremonte 1996).

El tercer estilo está conformado por pastas medias constituidas por inclusiones que representan entre $11 \%$ y $28 \%$ del total de la pasta y una granulometría media $(250 \mu \mathrm{m}$ a $1.000 \mu \mathrm{m})$, es decir, que se habrían usado arenas seleccionadas como atemperante (Figura 3d). Dentro de este estilo se registró una modalidad que se caracteriza por tener estructuras de fondo pseudolepidoblásticas con agregado de cristaloclastos de cuarzo, feldespato potásico y litoclastos graníticos. Una segunda está conformada por pastas que presentan los mismos atemperantes, pero la estructura de fondo es criptofilitosa. Finalmente, la tercera modalidad detectada reúne a pastas que presentan abundancia relativa de elementos no plásticos de origen volcánico, rocas posiblemente de la serie andesita, y vidrio volcánico. En las áreas aledañas se ha podido constatar cerámicas con inclusiones de origen volcánico en fragmentos procedentes del cementerio de La Ciénaga, valle de Hualfín (cortes 51 y 52, ver Zagorodny y Balesta 2005) y en las excavaciones de la aldea de Piedras Negras del oasis de Laguna Blanca (Espiro Elsesser 2006).

\section{Pastas finas}

Por otro lado, entre las pastas finas pudimos reconocer dos estilos técnicos. El primero está constituido por pastas que presentan entre $10 \%$ y $26 \%$ de elementos no plásticos de granulometría limo grueso a arena fina $(30 \mu \mathrm{m}$ a $250 \mu \mathrm{m})$, los que habrían sido incorporados intencionalmente. La mayoría de estas inclusiones corresponden a pequeños cristaloclastos de cuarzo (Figura 3e). El segundo estilo técnico está conformado por una serie de pastas elaboradas a partir de arcillas que poseen elementos no plásticos de forma natural. El material no plástico oscila entre $3 \%$ y $8 \%$ del total de la pasta. Es decir, que las alfareras y alfareros usaron arcillas sin agregarle ningún tipo de atemperante (Figura $3 \mathrm{f}$ ).

\section{Pastas Vaquerías}

Por último, se detectó un estilo de pastas que se corresponden exclusivamente a fragmentos del estilo polícromo Vaquerías. Son pastas medias con agregado de $20 \%$ a $25 \%$ de material no plástico de 
granulometría arena a arena gruesa $(500 \mu \mathrm{m}$ a 2.000 $\mu \mathrm{m})$. Los atemperantes detectados corresponden a litoclastos metamórficos (pizarra-filita), arenisca, litoclastos de cuarzo y tiesto molido (Figura $3 \mathrm{~g}$ ). Estas rocas metamórficas de bajo grado son materias primas no disponibles en el área de estudio, razón por lo que se postula que la cerámica Vaquerías fue una producción alóctona (Bugliani y Pereyra Domingorena 2012; Pereyra Domingorena 2010, 2012).

\section{Los Recipientes y Pastas Cerámicas}

Uno de los objetivos de la investigación fue vincular los patrones de pasta observados con las vasijas arqueológicas. La reconstrucción parcial de los recipientes permitió conocer el repertorio morfológico empleado en las prácticas cotidianas y periódicas de las antiguas sociedades aldeanas que habitaron el sur Calchaquí durante el primer milenio d.C. Sabemos que los pobladores prehispánicos usaban distintos tipos de ollas, cántaros, jarras, jarros, vasos, cuencos y escudillas (Bugliani 2008; Pereyra Domingorena 2010, 2012; Scattolin1990, 2007b).

\section{Cántaros y ollas}

En el conjunto cerámico analizado pudimos reconstruir cántaros y ollas subglobulares $(n=33)$ presentes a lo largo de toda la secuencia cronológica estudiada, es decir, a lo largo de las tres fases descritas por Scattolin (2007a). En general, estas piezas presentan las superficies alisadas, pero también hay ejemplares con superficies pulidas y muy pocas tienen diseños incisos. Estas cerámicas están asociadas a la cocción y almacenaje de alimentos (Bugliani 2008). El 63,6\% $(n=21)$ de estos recipientes fueron elaborados con pastas gruesas (Figura 4).

Finalmente, se registraron pequeñas ollas pulidas $(\mathrm{n}=4)$, consideradas como parte de la vajilla de servicio. Estas piezas fueron elaboradas con pastas finas sin atemperantes (olla 839-2 y olla 43-3) y con pastas finas con inclusiones no plásticas finas (olla 43-12 y olla 96-4).

Pero en los últimos siglos del primer milenio d.C. aparece un tipo de olla de cuerpo globular y cuello cilíndrico corto, terminado en borde recto horizontal sobresaliente (Scattolin 2007b). Es interesante notar que para elaborar este nuevo tipo de olla $(n=6)$ se utilizaron varios estilos técnicos. Por ejemplo, en el sitio Morro de las Espinillas se halló la olla 443-2, esta se manufacturó con una pasta gruesa (Figura 5a). En el componente Colalao del sitio Bañado Viejo se detectó la pieza 401-M8, que fue levantada con una pasta media con arenas no seleccionadas (Figura 5b). Otro caso es el ejemplar 454-6 encontrado en Morro de las Espinillas que fue

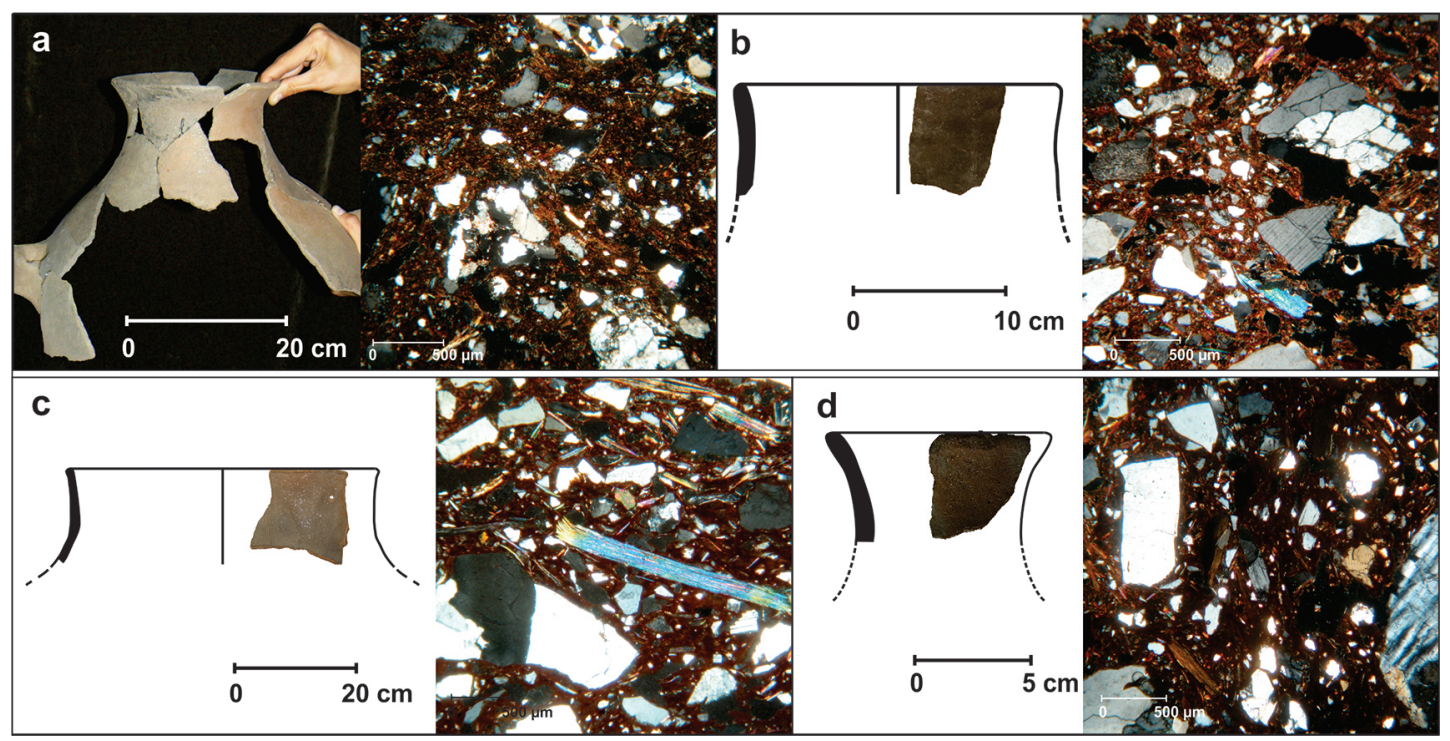

Figura 4. Ollas y cántaros de pasta gruesa: (a) Cántaro C65-T32 [Cardonal]; (b) Cántaro 461-R1 [Bañado Viejo]; (c) Olla 53-T9 [Loma Alta]; (d) Cántaro 369-10 [Loma Alta].

Pots and pitchers of coarse-grained paste: (a) pitcher C65-T32 [Cardonal]; (b) pitcher 461-R1 [Bañado Viejo]; (c) pot 53-T9 [Loma Alta]; (d) pitcher 369-10 [Loma Alta]. 
confeccionado con una pasta media con inclusiones grandes seleccionadas (Figura 5c). Finalmente, se quiere resaltar también que estas ollas se hicieron con el estilo técnico de pasta fina con el agregado de una arena muy fina (Figura 5d). Esta variabilidad en las estilos técnicos utilizados para elaborar este nuevo tipo morfológico puede ser explicado por la necesidad de nuevos bienes a causa de las nuevas configuraciones sociales que se gestaban en el Noroeste argentino (Scattolin 2007b).

\section{Jarras, jarros y vasos}

Otro conjunto de recipientes presente a lo largo de toda la secuencia cronológica estudiada está constituido por las jarras, los jarros y los vasos $(n=24)$. Son recipientes cerrados con cuellos largos y/o bien definidos donde el diámetro mínimo es superior o igual a un tercio del diámetro máximo (jarras) o de paredes verticales o levemente evertidas donde el diámetro de la abertura es igual o inferior a una vez y media la altura (jarros y vasos) (Bugliani 2008).

Principalmente para la manufactura de este conjunto de piezas se utilizaron pastas con arenas seleccionadas de granulometría media $(\mathrm{n}=11$, 47,8\%) (Figura 6a y b). En segundo término se empleó pastas finas con el agregado de arena fina como antiplástico $(\mathrm{n}=8,34,8 \%)$ (Figura $6 \mathrm{c}$ y d). También se pudo constatar otros casos donde se empleó pastas medias con arenas no seleccionadas (jarra 34-13, jarra 75-66 y jarro 134-SN) hallados en el contexto doméstico Núcleo E de Loma Alta (Figura 6e). Finalmente en el sitio Cardonal se halló una vasija efigie "quirquincho" (C70-T4) y una posible jarra (C54-T21) que presentan pasta fina sin agregado de inclusiones (Figura 6f).

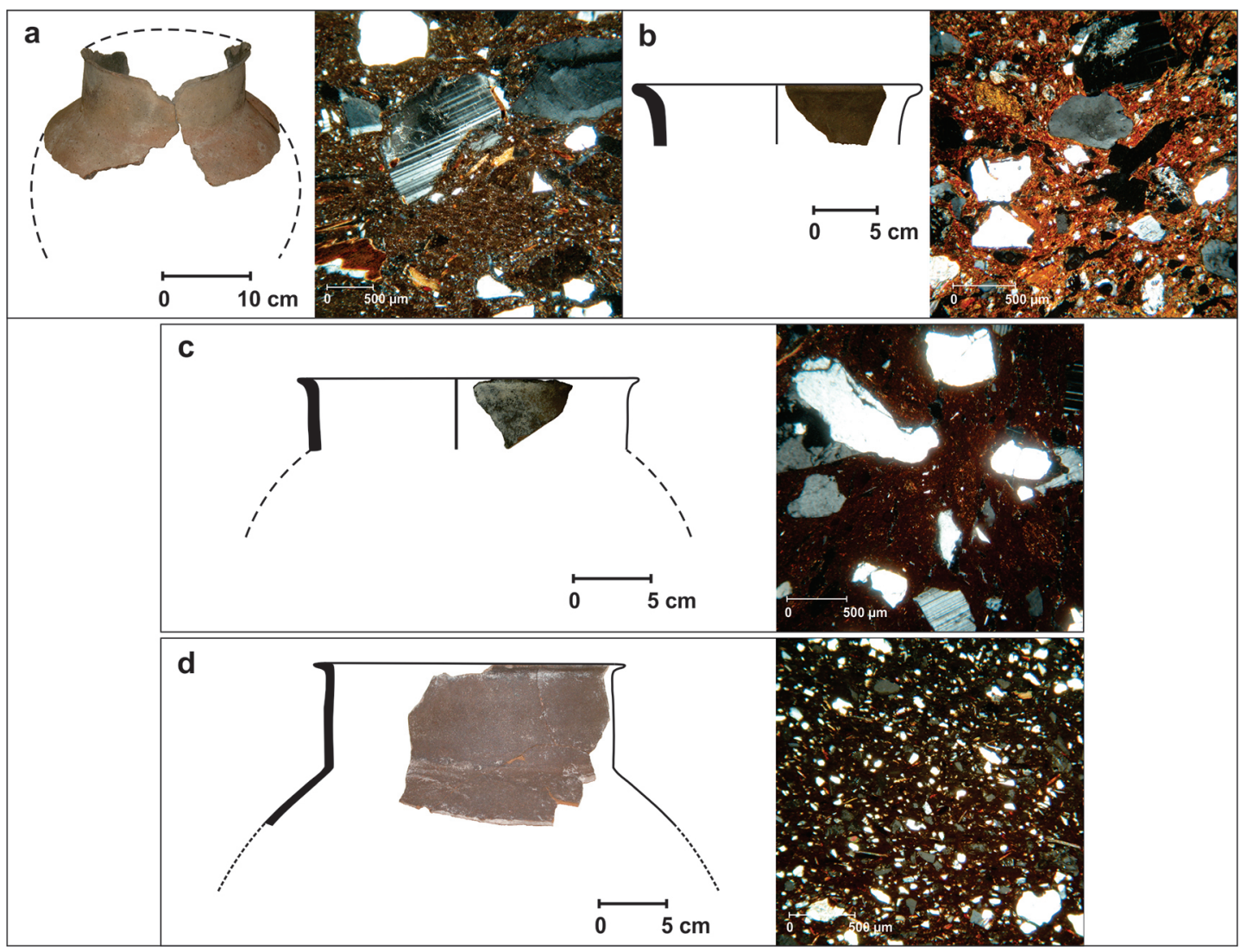

Figura 5. Ejemplos de distintos tipos de pastas en ollas con cuello cilíndrico. (a) 443-2 [Morro de las Espinillas]; (b) 401-M8 [Bañado Viejo]; (c) 454-6 [Morro de las Espinillas]; (d) 448-15 [Morro de las Espinillas].

Examples of different types of ceramic fabrics in pots with cylindrical necks: (a) 443-2 [Morro de las Espinillas]; (b) 401-M8 [Bañado Viejo]; (c) 454-6 [Morro de las Espinillas]; (d) 448-15 [Morro de las Espinillas]. 


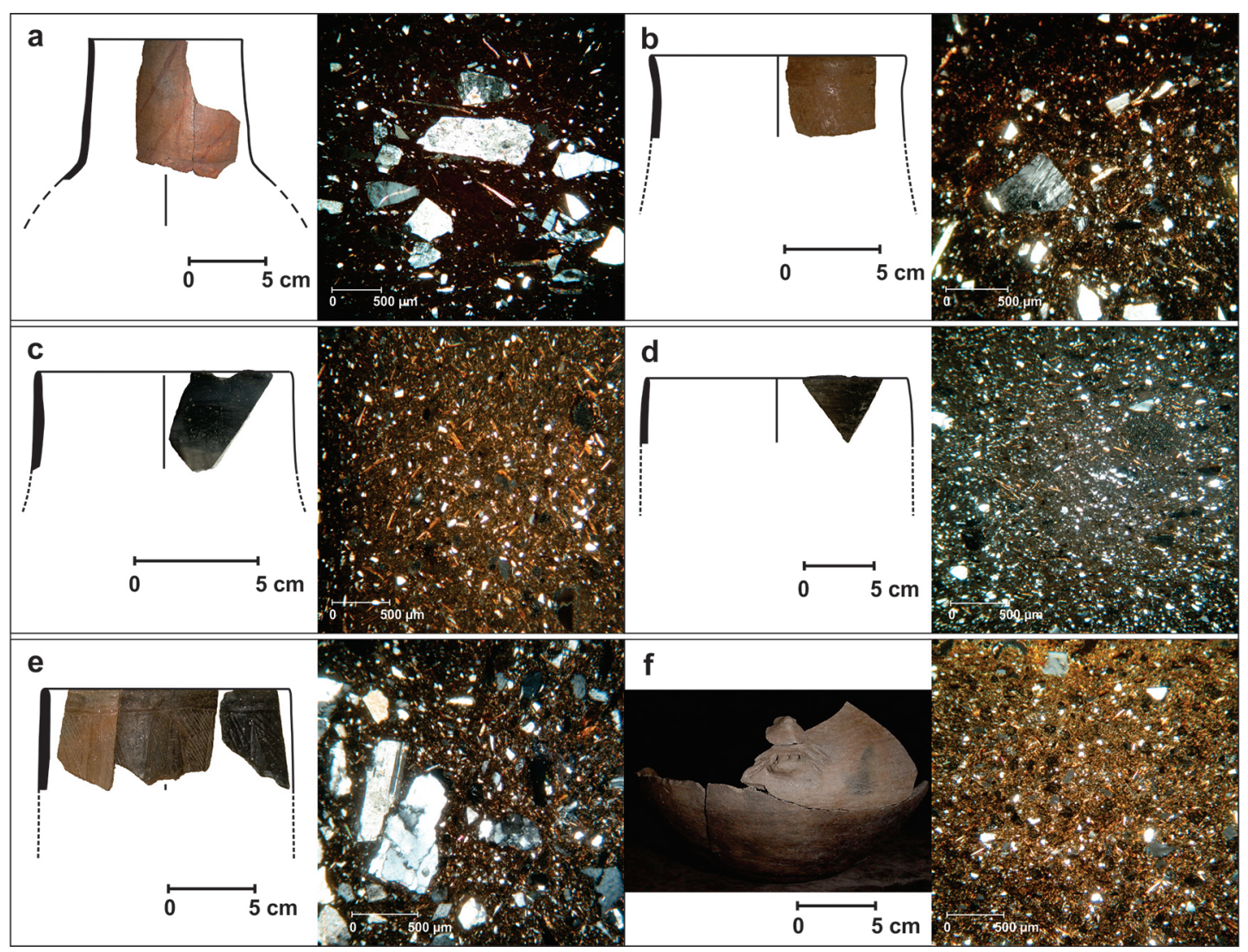

Figura 6. Ejemplos de distintos tipos de pastas en jarras, jarros y vasos: (a) jarra 541-9 [Antigal de Tesoro]; (b) jarra 378-11 [Loma Alta]; (c) jarra 473-1 [Bañado Viejo]; (d) jarro 584-1 [Tesoro 1]; (e) jarro 134-SN [Loma Alta]; (f) posible jarra C54-T21 [Cardonal]. Examples of different types of ceramic fabrics in jugs, mugs and beakers: (a) jug 541-9 [Antigal de Tesoro]; (b) jug 378-11 [Loma Alta]; (c) jug 473-1 [Bañado Viejo]; (d) mug 584-1 [Tesoro 1]; (e) mug 134-SN [Loma Alta]; (f) possible jug C54-T21 [Cardonal].

Es interesante destacar que este conjunto de formas no aparecen asociadas a las pastas gruesas con las que se hicieron la mayoría de las ollas y los cántaros.

\section{Cuencos y escudillas}

Un tercer gran conjunto de recipientes está representado por los cuencos y las escudillas $(n=41)$. Estos son vasijas abiertas, en el caso de los cuencos el diámetro de la abertura (boca) está comprendido entre una vez y media y dos veces y media la altura. En cambio, las escudillas se distinguen porque presentan diámetros que varían entre dos veces y media y cinco veces la altura (Bugliani 2008). Más de la mitad de las piezas $(\mathrm{n}=24,58,5 \%)$ fueron elaborados con el estilo técnico de pasta fina con agregado de arenas muy finas (Figura 7a y b). Además se detectaron cuecos y escudillas confeccionados con pastas finas sin agregado de inclusiones $(\mathrm{n}=12,29,3 \%)$ (Figura 7c).

Debemos destacar igualmente la presencia de casos particulares de escudillas grandes (137-45 y 137-46) que se elaboraron con pastas gruesas (Figura 7d), es decir, ese estilo técnico usado para hacer ollas y cántaros. Estas piezas fueron halladas en el contexto doméstico Núcleo E de Loma Alta. Es interesante remarcar que pucos de pastas gruesas se han encontrado en las excavaciones de Yutopián (Bugliani 2008) y en los sitios formativos de la quebrada de La Ciénega (Cremonte 1996). Finalmente cabe mencionar la presencia de la Escudilla 478-3 y el Cuenco 185-4 de pasta media poco seleccionada (Bañado Viejo y Loma Alta, respectivamente) (Figura 7e) y el Cuenco 448-17 de pasta con inclusiones de tamaños medios seleccionados, de Morro de las Espinillas (Figura 7f). 


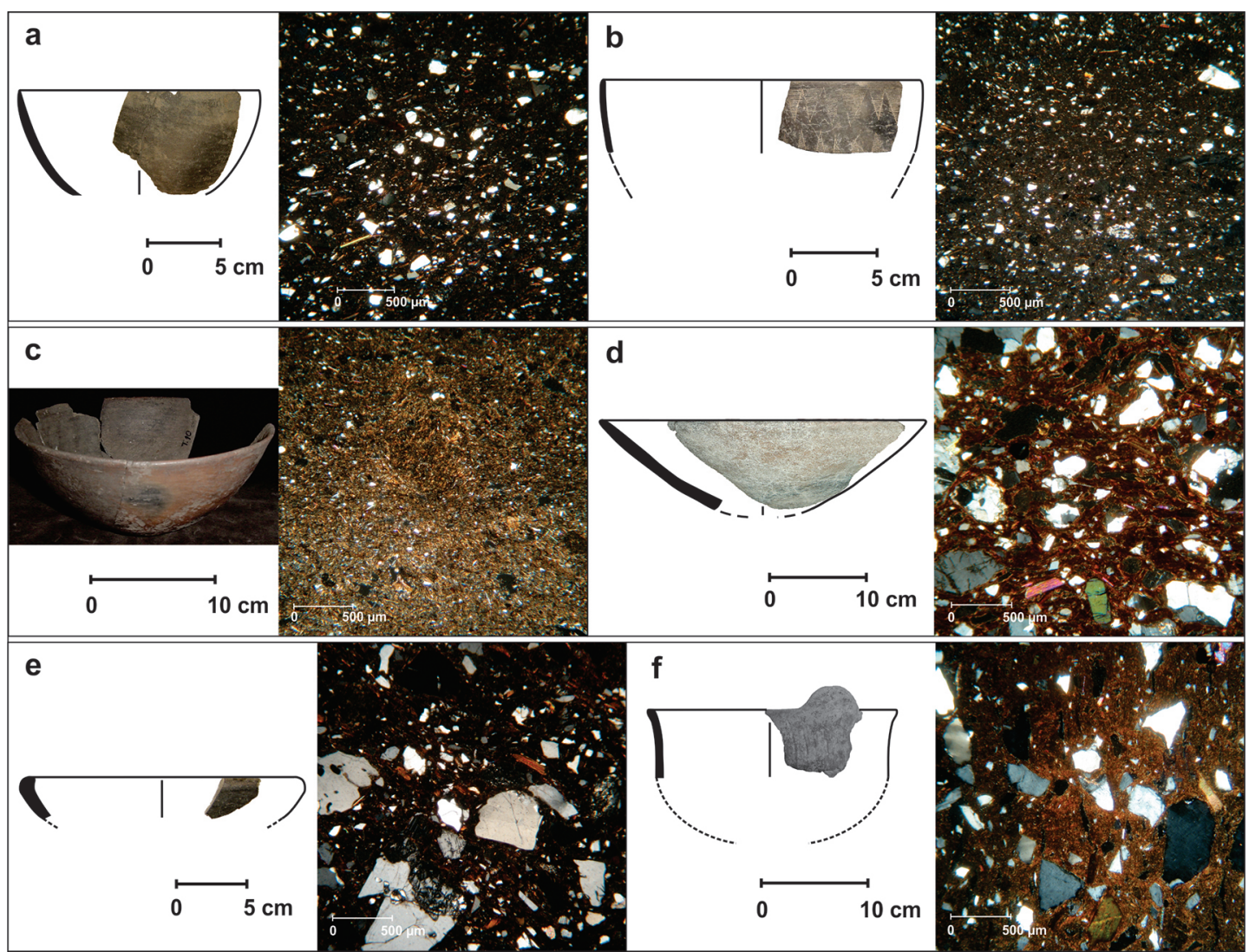

Figura 7. Ejemplos de distintos tipos de pastas en cuencos y escudillas: (a) cuenco 467-12 [Bañado Viejo]; (b) cuenco 560-T3 [Tesoro 1]; (c) cuenco C70-T10 [Cardonal]; (d) escudilla 478-3 [Bañado Viejo]; (e) cuenco 185-4 [Loma Alta]; (f) cuenco 448-17 [Morro de las Espinillas].

Examples of different types of ceramic fabrics in bowls and shallow bowls: (a) bowl 467-12 [Bañado Viejo]; (b) bowl 560-T3 [Tesoro 1]; (c) bowl C70-T10 [Cardonal]; (d) shallow bowl 478-3 [Bañado Viejo]; (e) bowl 185-4 [Loma Alta]; (f) bowl 448-17 [Morro de las Espinillas].

\section{Estudios Experimentales}

Por otra parte se realizaron estudios experimentales con arcillas $(n=5)$ y arenas $(n=3)$ recolectadas en el área de estudio con el objetivo de evaluar por otras vías los patrones de pastas que observábamos en el estudio petrográfico de las cerámicas arqueológicas.

Dos de las arcillas provienen del sur del valle del Cajón, una de la falda occidental del Aconquija, otra del fondo de valle de Santa María y la quinta del sur de la quebrada de Las Conchas (Pereyra Domingorena 2013:Figura1). Las arcillas fueron localizadas con dos criterios. Por un lado se recogió información etnográfica en el sur del valle del Cajón. En el pasado reciente nuestros informantes elaboraban vasijas tanto para el consumo doméstico como para la venta (Calo y Pereyra Domingorena 2013). También se entrevistó a un artesano de la ciudad de Santa María (Catamarca), quien produce cerámica para el consumo turístico. El segundo criterio fue la lectura de las cartas geológicas del área, las que permitieron ubicar algunas potenciales fuentes de arcilla de la región.

Las arenas recolectadas provienen de las cercanías de los lugares donde se recogieron los materiales arcillosos. Una de ellas fue colectada de un lecho seco de un pequeño arroyo localizado en el faldeo occidental del Aconquija. Otra muestra fue recogida en el lecho del río Santa María. Finalmente, la tercera de las arenas corresponde a una muestra procedente del lecho del río Las Conchas (Pereyra Domingorena 2013:Figura 1). 
La preparación de las muestras consistió en sumergir las arcillas en agua para su hidratación y la extracción manual de la materia orgánica y los grandes clastos. Posteriormente fueron amasadas en estado plástico y fueron agregadas las inclusiones en las series que así lo requerían. Las series se confeccionaron usando una determinada arcilla, a la que, según el caso, se le agregó diferentes proporciones de una arena recogida en las cercanías de los depósitos arcillosos.

La Serie 1 y la Serie 2 fueron confeccionadas con las arcillas del sur del valle del Cajón y no se agregó material atemperante. En la Serie 3 se utilizaron la arcilla y arena recolectadas en la falda del Aconquija. Una parte de la misma se confeccionó solo con arcilla, es decir, no se agregó ningún tipo de inclusiones. Con otra parte se preparó una mezcla de arcilla con $15 \%$ de arena. Esta fue tamizada con un cernidor de 250 $\mu \mathrm{m}$. El objetivo fue replicar un patrón observado en la cerámica arqueológica que correspondía a pastas con agregado intencional de material no plástico fino. Finalmente, se combinó arcilla con arena al $40 \%$ sin cernir, o sea, agregando el atemperante tal cual fue recogido. En este caso el fin fue reproducir el patrón registrado en las pastas gruesas usadas para manufacturar ollas y cántaros en el pasado prehispánico (Pereyra Domingorena 2010, 2013).

La Serie 4 fue confeccionada con la arcilla y la arena recogidas en el valle de Santa María. Finalmente, la Serie 5 fue elaborada con la arcilla y la arena colectadas en la quebrada de Las Conchas. Para ambas se siguió el mismo proceso de elaboración descrito para la Serie 3.

Las muestras experimentales fueron cocidas en horno eléctrico con control automático de temperatura. El tiempo de cocción aproximadamente fue de 1 hora 30 minutos para alcanzar la temperatura requerida; 10 minutos de cocción a esa temperatura; y 24 horas de enfriamiento dentro del horno. La intención fue recrear los picos de temperaturas registrados en algunas experiencias etnográficas y experimentales donde se han medido las temperaturas de las cocciones (Cremonte 1989-1990, 1996; García 1993; Palamarczuk 2004). Los cortes delgados utilizados para la comparación con las pastas arqueológicas provienen de las muestras cocidas a $650^{\circ}$ de las distintas series experimentales. Esta decisión se fundamentó a partir de las similitudes texturales registradas - por medio de lupa binocular- entre las muestras arqueológicas y experimentales (Pereyra Domingorena 2010, 2013).

Los análisis petrográficos de las secciones delgadas de las briquetas experimentales permitieron evaluar tres estilos técnicos de manufactura registrados en la cerámica arqueológica del área: pastas finas sin agregado de atemperantes, pastas finas con agregado de inclusiones y pastas gruesas.

Las muestras experimentales confeccionadas con arcillas sin agregado de inclusiones no plásticas registraron el predominio de la matriz arcillosa con escaso contenido de material no plástico natural -menos del 5\%-, y estructuras de fondo de pasta pseudolepidoblástica algo criptofilitosa (Pereyra Domingorena 2013:Figura 7a, b, c y d). El uso de arcillas sin inclusiones como patrón técnico fue detectado en la cerámica arqueológica (Figuras 3f; 6f; 7c). Las similitudes en las estructuras de fondo de la matriz, cualitativas y cuantitativas de ambos conjuntos cerámicos -experimental y arqueológicofortalecieron la hipótesis sobre la existencia en el pasado prehispánico de un estilo técnico que implicó el uso de arcillas con escasísimo contenido de inclusiones naturales (Pereyra Domingorena 2010, 2013).

En cambio, las briquetas experimentales con agregado intencional del 15\% de arenas tamizadas (Pereyra Domingorena 2013:Figura 8a y b) no lograron generar el patrón registrado en las pastas finas arqueológicas que presume el uso de una arena muy fina como atemperante (Figuras 3e; $5 d ; 6 c$ y d; 7a y b). Sin embargo, la existencia del estilo técnico fue corroborada por otros estudios cerámicos del Noroeste argentino (Cremonte et al. 2003; Palamarczuk 2009).

Finalmente, la comparación entre muestras experimentales con agregado intencional de material no plástico (Pereyra Domingorena 2013:Figura 9a, b y c) y las pastas gruesas arqueológicas (Figuras 3a; 4a, b, c y d; 5a; 7d) permitió resaltar las semejanzas de ambos conjuntos; particularmente en relación con las estructuras de fondo pseudolepidoblásticas y con la granulometría y porcentaje de las inclusiones no plásticas (Pereyra Domingorena 2010, 2013).

\section{Comentarios Finales}

De esta manera, esta investigación procuró hilvanar distintos niveles de análisis poniendo en relieve a los recipientes cerámicos hallados en los contextos de excavación de los sitios arqueológicos 
estudiados. Se logró vincular la morfología de los mismos, los tipos de pastas y los atributos de diseño. Así, se determinaron seis patrones técnicos asociados a producciones locales en el área de estudio y un estilo técnico asociado a una producción alóctona. Además, la experimentación con arcillas y arenas locales aportó una vía independiente de corroboración de algunos de los estilos técnicos alfareros prehispánicos postulados en esta investigación. De esta forma se comprobó la existencia de una tradición artesanal con manifestaciones locales que estuvo vigente durante casi la totalidad del primer milenio d.C. al sur de los valles calchaquíes.
Agradecimientos: A Emily Stovel y Beatriz Cremonte por la invitación a participar en el Simposio "Interactuemos también nosotros: el estado de la investigación cerámica en el Centro Sur Andino" organizado en el marco del XIX Congreso Nacional de Arqueología Chilena (Arica, octubre 2012). Dicha presentación se financió por medio del ANPCyTPICT 2007 Raíces No 116 y el CONICET-PIP 2009 $\mathrm{N}^{\circ} 256$, subsidios dirigidos por M. Cristina Scattolin. Mi reconocimiento a los evaluadores anónimos por los comentarios sugeridos que permitieron mejorar el manuscrito. No obstante, el contenido del mismo es de mi responsabilidad.

\section{Referencias Citadas}

Adams, A.E., W.S. Mackenzie y C. Guilford 1997 [1984]. Atlas de Rocas Sedimentarias. Masson, Barcelona.

Balfet, H., M.F. Fauvet-Berthelot y S. Monzón 1983. Pour la Normalisation de la Description des Poteries. Editions du Centre National de la Recherche Scientifique, Paris.

Balfet, H., M.F. Fauvet-Berthelot y S. Monzón 1988. Lexique Plurilingue pour la Description des Poteries. Editions du Centre National de la Recherche Scientifique, Paris.

Bugliani, M.F. 2008. Consumo y Representación en el Sur de los Valles Calchaquíes (Noroeste Argentino). Los Conjuntos Cerámicos de las Aldeas del Primer Milenio. John and Erica Hedges, Oxford.

Bugliani, M.F. y L. Pereyra Domingorena 2012. Una aproximación estilístico-tecnológica a la cerámica polícroma "Vaquerías" del Noroeste Argentino. Estudios Atacameños 43:121-138.

Calo, C.M. y L. Pereyra Domingorena 2013. El ambiente y los recursos: un estudio sobre la recolección en La Quebrada (Catamarca, Argentina). Revista Española de Antropología Americana 43:133-154.

Cremonte, M.B. 1989-1990. La alfarería tradicional actual: reflexiones y posibles aplicaciones para la arqueología a través de dos casos de estudio. Runa XIX:117-133.

Cremonte, M.B. 1996. Investigaciones Arqueológicas en la Quebrada de La Ciénega (Dpto. de Tafi, Tucumán). Tesis para optar al grado de Doctor en Ciencias Naturales, Mención Arqueología, Facultad de Ciencias Naturales y Museo, Universidad Nacional de La Plata, La Plata.

Cremonte, M.B., M. Baldini e I.L. Botto 2003. Pastas y colores. Un camino al conocimiento del estilo Portezuelo de Aguada. Intersecciones en Antropología 4:3-16.

Courtois, L. 1976. Examen au Microscope Pétrographique des Céramiques Archéologiques. Centre National de la Recherche Scientifique, Paris.

Culbert, P. y R.L. Rands 2007. Multiple classifications: an alternative approach to the investigation of Maya ceramics. Latin American Antiquity 18:181-190.
Espiro Elsesser, V.E. 2006. Aportes para una Clasificación Tecnológica de las Cerámicas Pertenecientes al primer Milenio de nuestra era de la aldea Piedra Negra, Laguna Blanca, Dpto. Belén, Provincia de Catamarca. Tesis para optar al grado de Licenciado, Mención Arqueología. Escuela de Arqueología, Universidad Nacional de Catamarca, San Fernando del Valle de Catamarca.

Galván, A. 1981. Descripción Geológica de la Hoja 10e, Cafayate. Servicio Geológico Nacional, Buenos Aires.

García, L.C. 1993. Experimentación en Inca Cueva: arcillas, fogones y combustibles. Arqueología 3:69-91.

González Bonorino, F. 1951. Descripción Geológica de la Hoja 12e, Aconquija. Dirección Nacional de Minería, Buenos Aires.

Gosselain, O.P. 2008. Thoughts and adjustments in the potter's backyard. En Breaking the Mould: Challenging the Past through Pottery, editado por I. Berg, pp. 67-79. Archaeopress, Oxford.

Haber, A. 2007. Comentarios marginales. En Sociedades Precolombinas Surandinas. Temporalidad, Interacción y Dinámica Cultural del NOA en el Ámbito de los Andes CentroSur, editado por V. Williams, B. Ventura, A. Callegari y H. Yacobaccio, pp. 59-72. Instituto de Arqueología-Facultad de Filosofía y Letras-Universidad de Buenos Aires, Buenos Aires.

Hammer, Ø., D.A.T. Harper y P.D. Ryan 2001. PAST: Palaeontological statistics software package for education and data analysis. Palaeontologia Electronica 4:1-9.

Kerr, P.F. 1965. Mineralogía Óptica. McGraw-Hill Book Company, Nueva York.

Klein, C. y C.S. Hurlbut Jr. 1998. Manual de Mineralogía. Editorial Reverté, Barcelona.

Lemonnier, P. 1992. Elements for Anthropology of Technolog. Museum of Anthropology-University of Michigan, Ann Arbor.

Linné, S. 1925. The Technique of South American Ceramics. Elanders Boktryckeri Aktieboi, AG, Gotemburgo.

Mauss, M. 1979 [1935]. Técnicas y movimientos corporales. En Sociología y Antropología, pp. 337-356. Editorial Tecnos, Madrid. 
Palamarczuk, V. 2004. Cocción experimental de cerámica con estiércol de llama. Intersecciones en Antropología 5:119-127.

Palamarczuk, V. 2009. Un Estilo y su Época. El Caso de la Cerámica Famabalasto Negro Grabado del Noroeste Argentino. Tesis para optar al grado de Doctor en Arqueología, Facultad de Filosofía y Letras, Universidad de Buenos Aires, Buenos Aires.

Pereyra Domingorena, L. 2010. Manufacturas Alfareras de las Sociedades Aldeanas del Primer Milenio d.C. al Sur de los Valles Calchaquíes. Tesis para optar al grado de Doctor en Arqueología, Facultad de Filosofía y Letras, Universidad de Buenos Aires, Buenos Aires.

Pereyra Domingorena, L. 2012. Manufacturas alfareras al sur de los valles Calchaquíes entre el siglo primero al quinto D.C. Relaciones de la Sociedad Argentina de Antropología 37:387-412.

Pereyra Domingorena, L. 2013. Cerámica experimental con materias primas del sur de los valles Calchaquíes (Noroeste argentino). Intersecciones en Antropología 14:239-250.

Puente, V. 2011. Prácticas de Producción Alfarera en el Valle del Bolsón (Belén, Catamarca). Materias Primas y Modos de Hacer ca. 900-1600 d.C. Tesis para optar al grado de Doctor en Arqueología, Facultad de Filosofía y Letras, Universidad de Buenos Aires, Buenos Aires.

Ruiz Huidobro, O.J. 1972. Descripción Geológica de la Hoja 11e, Santa María. Servicio Nacional Minero Geológico, Buenos Aires.

Scattolin, M.C. 1990. Dos asentamientos formativos al pie del Aconquija. El sitio Loma Alta (Catamarca, Argentina). Gaceta Arqueológica Andina V:85-100.
Scattolin, M.C. 2007a. Santa María antes del año mil. Fechas y materiales para una historia cultural. En Sociedades Precolombinas Surandinas: Temporalidad, Interacción y Dinámica Cultural del NOA en el Ámbito de los Andes Centro-Sur, editado por V. Williams, B. Ventura, A. Callegari y H. Yacobaccio, pp. 203-219. Instituto de Arqueología-Facultad de Filosofía y Letras-Universidad de Buenos Aires, Buenos Aires.

Scattolin, M.C. 2007b. Estilos como recursos en el Noroeste argentino. En Procesos Sociales Prehispánicos en el Sur Andino: La Vivienda, la Comunidad y el Territorio, compilado por A. Nielsen, C. Rivolta, V. Seldes, M. M. Vázquez y P. Mercolli, pp. 291-321. Brujas, Córdoba.

Stark, M.T. 1999. Social dimensions of technical choice in Kalinga ceramic traditions. En Material Meanings. Critical Approaches to the Interpretation of Material Culture, editado por E.S. Chilton, pp. 24-43. The University of Utah Prees, Salt Lake City.

Tarragó, M.N. 2007. Ámbitos domésticos y de producción artesanal en el Noroeste Argentino prehispánico. Intersecciones en Antropología 8:87-100.

Turner, J.C.M. 1973. Descripción Geológica de la Hoja 11d, Laguna Blanca. Servicio Nacional Minero Geológico, Buenos Aires.

Yardley, B.W.D. 1989. An Introduction to Metamorphic Petrology. Longman Scientific \& Technical, Harlow.

Zagorodny, N. y B. Balesta 2005. Estudio Multidimensional de la alfarería de La Ciénaga. En Azampay: Presente y Pasado de un Pueblito Catamarqueño, editado por M.C. Sempé, S. Salceda y M. Maffia, pp. 267-288. Ediciones Al Margen, La Plata.

\section{Notas}

1 Para la identificación mineralógica se utilizaron los libros: An introduction to metamorphic petrology (Yardley 1989), Atlas de rocas sedimentarias (Adams et al. 1997 [1984]), Manual de Mineralogía (Klein y Hurlbut Jr. 1998) y Mineralogía Óptica (Kerr 1965).

2 Para el análisis de conglomerados se usó el programa PAST versión 1.90 (Hammer et al. 2001).

3 En el caso concreto de las manufacturas alfareras prehispánicas aquí analizadas, las fuentes de energía consideradas son aquellas que fueron utilizadas en la cocción de los recipientes cerámicos. Las investigaciones experimentales y etnoarqueológicas proponen que leñas y/o excrementos de animales se pudieron haber empleado como combustible (Cremonte 1989-1990, 1996; Garcia 1993; Palamarzuck 2004). Además, debería considerarse la hipótesis del uso de marlos de maíz, ya que se demostró su consumo como combustible en la producción metalúrgica prehispánica del Noroeste argentino (Tarragó 2007). 